\title{
Psicooncología
}

ISSN: $1696-7240$

\section{Validación de un Cuestionario Multidimensional de Adaptación a la Enfermedad para Pacientes Renales en Diálisis (CMAE-RD) diseñado a partir de un cuestionario para pacientes oncohematológicos}

\author{
Rocío Rodríguez Rey ${ }^{*}$; Helena García-Llana²; José Luis Górriz ${ }^{3}$; Rafael Selgas ${ }^{4}$
}

Recibido; 10 de enero de 2020 / Aceptado: 1 de marzo de 2020

Resumen: Objetivos: El objetivo de este trabajo es estudiar las propiedades psicométricas de un cuestionario multidimensional de adaptación a la enfermedad para pacientes con enfermedad renal en diálisis (CMAE-RD).

Métodos: Esta herramienta está diseñada para ser administrada por un profesional de la psicología en forma de entrevista semiestructurada y fue diseñado a partir de un cuestionario previo para pacientes oncohematológicos. Un total de 113 pacientes en hemodiálisis fueron entrevistados mediante el CMAERD y completaron dos cuestionarios adicionales con propósitos de validación: el HADS (para evaluar ansiedad y depresión) y el CDRISC-2 (para evaluar resiliencia).

Resultados: La consistencia interna para las áreas del CMAE-RD estuvo comprendida entre 0,53 y 0,70, y se obtuvieron evidencias de validez relacionada con un criterio externo y concurrente especialmente para las áreas que evaluaban el grado en que el paciente está informado y su estado de ánimo.

Conclusiones: CMAE-RD presenta niveles adecuados de fiabilidad y validez, siendo una herramienta útil desde el punto de vista clínico, pues permite evaluar las necesidades y recursos de los pacientes, guiando la intervención psicológica.

Palabras clave: Enfermedad renal crónica avanzada; hemodiálisis; adaptación psicológica; evaluación psicológica.

1 Rocío Rodríguez-Rey. Departamento de Psicología. Facultad de Ciencias Humanas y Sociales. Universidad Pontificia Comillas. Madrid

E-mail: rocio.rodriguez.rey@gmail.com

2 Helena García-Llana. Servicio de Nefrología. Hospital Universitario La Paz-IdiPAZ; División Psicología, Centro de Enseñanza Superior Cardenal Cisneros; Departamento de Psicología, Facultad de Ciencias Humanas y Sociales. Facultad Universidad Pontificia Comillas.

E-mail: helenagllana@hotmail.com

3 José Luis Górriz. Servicio de Nefrología. Hospital Clínico Universitario. INCLIVA. Valencia. Universidad de Valencia.

E-mail: jlgorriz@senefro.org.

4 Rafael Selgas. Servicio de Nefrología, Hospital Universitario La Paz-IdiPAZ. Universidad Autónoma de Madrid; Instituto Reina Sofía de Investigaciones, Nefrológicas (IRSIN).

E-mail: rafael.selgas@salud.madrid.org

* Dirección de correspondencia: Rocío Rodríguez Rey. Departamento de Psicología. Facultad de Ciencias Humanas y Sociales. C/ Universidad Comillas 328049 - Madrid. E-mail: rocio.rodriguez.rey@gmail.com; rocio.r.rey@comillas.edu 


\title{
[en] Validation of a Multidimensional Questionnaire to Assess Adaptation to Illness to Renal Patients under Dialysis Treatment designed from a questionnaire from oncohaematologic patients
}

\begin{abstract}
Objectives: The present study aims to explore the psychometric properties of a multidimensional questionnaire to assess adaptation to illness in patients with end stage renal disease under hemodialysis (CMAE-RD for its Spanish acronym).

Methods: This instrument was developed to be administered by a psychologist as a semi structured interview and it was adapted from a previous questionnaire for patients with oncohaematologic diseases. A total of 113 patients receiving hemodialysis treatment were interviewed with the CMAE$\mathrm{RD}$ and completed two additional questionnaires with validation purposes: the HADS (to assess anxiety and depression) and the CDRISC-2 (to assess resilience).

Results: Internal consistency scores for the areas of the CMAE-RD was comprised between.53 and.70. Evidences of validity related to an external criterion and concurrent validity were obtained for the areas which assess the level of information of the patient and their emotional state.

Conclusions: We conclude that the CMAE-RD shows adequate levels of reliability and validity, being a useful measurement tool from the standpoint of health care, as it allows psychologist the needs and resources of renal patients, providing guidance for psychological intervention.
\end{abstract}

Keywords: Advanced chronic kidney disease; hemodialysis; psychological adjustment; psychological assessment.

Sumario: 1. Introducción 2. Método 3. Resultados 4. Discusión 5. Conclusiones 6. Agradecimientos 7. Referencias bibliográficas

Cómo citar: Rodríguez Rey R; García-Llana H; Górriz JL; Selgas R. Validación de un Cuestionario Multidimensional de Adaptación a la Enfermedad para Pacientes Renales en Diálisis (CMAE-RD) diseñado a partir de un cuestionario para pacientes oncohematológicos. Psicooncología 2020;17:149164. doi: $10.5209 /$ psic.68247.

\section{Introducción}

El presente trabajo tiene como objetivo el estudio de las propiedades psicométricas de un cuestionario multidimensional de adaptación a la enfermedad para pacientes con enfermedad renal crónica avanzada (ERCA) en tratamiento de diálisis. Enfermedad renal crónica (ERC) es un término general que se utiliza para referirse a un conjunto heterogéneo de trastornos que afectan a la estructura y función de los riñones ${ }^{(1)}$. La enfermedad avanza a través de 5 estadios en función de la gravedad del fallo renal, considerándose en los dos últimos que la persona se encuentra en situación de ERCA. En España, la prevalencia de la ERC es del 15,1\%, afectando a uno de cada 7 adultos ${ }^{(2)}$.

Cuando el paciente está en situación de ERCA, requiere tratamiento renal sustitutivo (TRS). Las opciones de TRS son trasplante renal o diálisis. El trasplante se asocia con una mayor supervivencia a largo plazo que la diálisis, pero se encuentra limitado por la escasez de donantes ${ }^{(1)}$. Existen dos modalidades de diálisis: hemodiálisis (HD) y diálisis peritoneal. La HD es el tratamiento más habitual, y suele realizarse en el hospital o en centros específicos de diálisis. La HD conlleva una elevada carga de síntomas, como sequedad cutánea, fatiga, picores o dolor articular u óseo, lo que se encuentra directamente correlacionado con un empeoramiento de 
la calidad de vida, y con la presencia de sintomatología depresiva ${ }^{(3)}$. A la carga de síntomas se unen habitualmente otros estresores, como regímenes terapéuticos muy complejos, o una frecuente falta de ocupación laboral ${ }^{(4,5)}$. Todo ello conduce a un alto riesgo de sufrir alteraciones emocionales ${ }^{(6,7)}$. La tasa de depresión en distintos estudios oscila entre el 35 y el $44 \%{ }^{(8,9)}$, encontrándose una prevalencia de trastornos de ansiedad del $45,7 \%{ }^{(10)}$.

La atención sanitaria del siglo XXI debe tener como objetivo aliviar el sufrimiento de los pacientes, y no solo tratar sus enfermedades ${ }^{(11)}$. Para lograrlo es necesario que entre los cuidados rutinarios del paciente con ERCA se incluya la atención a los aspectos psicológicos, espirituales y sociales ${ }^{(12,13)}$. Esto hace necesario que los profesionales sanitarios que trabajan en contacto con el paciente renal dispongan de herramientas específicas de evaluación de su estado emocional ${ }^{(14)}$. Un avance importante en este sentido ha sido el desarrollo del instrumento de Evaluación del Estado Emocional para pacientes Renales en Diálisis (EE-D), una herramienta de screening específicamente diseñada para que el personal de enfermería monitoree el estado emocional de sus pacientes ${ }^{(15,16)}$.

La monitorización del estado emocional de los pacientes en diálisis por parte del personal sanitario, y el apoyo emocional, aunque necesarios, no son suficientes para atender con el máximo grado de calidad asistencial a los pacientes con ERCA. La prevalencia y complejidad de las dificultades psicológicas que pueden aparecer en estos pacientes, frecuentemente exceden las competencias de la intervención médica y de enfermería, lo que hace necesaria la incorporación de la figura del psicólogo como parte del equipo interdisciplinar de las unidades de Nefrología ${ }^{(17,18)}$. En este contexto, es necesario que los psicólogos que trabajan en estas unidades dispongan de herramientas específicas de evaluación psicológica.

Para facilitar la evaluación psicológica de las necesidades y recursos relacionados con la adaptación a la enfermedad del paciente con ERCA, Rodríguez-Rey et al. ${ }^{(19)}$ desarrollaron el Cuestionario Multidimensional de Adaptación a la Enfermedad para pacientes Renales en Diálisis (CMAE-RD) a partir de una adaptación del Cuestionario Multidimensional de Adaptación a la Enfermedad para pacientes Onco hematológicos ${ }^{(20)}$. Esta herramienta evalúa necesidades y recursos del paciente renal, que se relacionan con su adaptación a la ERCA. Se basa en los modelos teóricos desarrollados por Costa y López ${ }^{(21)}$, que a su vez se basa en los trabajos de Albee ${ }^{(22)}$ y Lazarus y Folkman ${ }^{(23)}$. Este enfoque tiene en cuenta no sólo las necesidades de los pacientes, de cara a cubrirlas, sino también sus recursos y habilidades con el propósito de potenciarlos.

El artículo de Rodríguez-Rey et al. ${ }^{(19)}$ que describe el desarrollo de la herramienta CMAE-RD se compuso de tres estudios secuenciales. El primero de ellos fue un estudio interjueces para determinar la validez del cuestionario, el segundo un estudio piloto para poner a prueba su funcionamiento, y el tercero un estudio de validación con una muestra de 54 pacientes. Estos estudios permitieron depurar el CMAE$\mathrm{RD}$ y mostraron que es una herramienta útil y adecuada para evaluar necesidades y recursos relacionados con la adaptación a la enfermedad en pacientes en diálisis. No obstante, antes de poder utilizarlos con las garantías suficientes es necesaria la realización de un estudio con una muestra mayor y empleando la versión definitiva para conocer sus propiedades psicométricas en términos de fiabilidad (consistencia interna) y validez convergente (relaciones con otras variables). La realización de este estudio es el objetivo del presente artículo. Esperamos que, al ser utilizado 
con una muestra mayor, el CMAE-RD muestre indicadores de consistencia interna similares a las del estudio original de Rodríguez-Rey ${ }^{(19)}$. Con respecto a la relación de los ítems del CMAE-RD con variables clínicas y sociodemográficas, esperamos lo siguiente:

- Que las personas con un mayor índice de comorbilidad sean aquellas que tengan menor conocimiento y comprensión la información médica, y sean menos autónomos en la toma de la medicación.

- Que los pacientes con peor pronóstico evaluado por su médico muestren niveles mayores de tristeza y ansiedad, así como menor control percibido.

- Esperamos que las personas con historia psicopatológica muestren menores niveles de adherencia y mayores niveles de tristeza y ansiedad.

- Que los pacientes de mayor edad estén menos informados y entiendan menos la información sobre la enfermedad y tratamientos. Además, esperamos que tengan un menor deseo de participar en la toma de decisiones sobre su enfermedad y tratamientos, que perciban que participan menos y que sean menos autónomos en la toma de medicación.

- Que las personas con mayor nivel de estudios estén más informadas.

Con respecto a la relación de los ítems del CMAE-RD con las puntuaciones en cuestionarios que miden variables relacionadas (ansiedad, depresión y resiliencia), nuestra expectativa general es que las correlaciones aportarán evidencias de validez convergente para las puntuaciones del CMAE-RD. En concreto, nuestras hipótesis son:

- Esperamos que los dos ítems del área "Estado de ánimo" se asocien positivamente con las puntuaciones en ansiedad y depresión. En concreto, esperamos que el ítem del CMAE-RD que evalúa ansiedad, se asocie más fuertemente con ansiedad que con depresión, y viceversa para el ítem que evalúa tristeza.

- Esperamos que los dos ítems del área "Estado de ánimo" correlacionen negativamente con resiliencia.

- Con respecto al apoyo social, dado que es un reconocido factor protector, esperamos que se asocie con mayor resiliencia y menor ansiedad y depresión.

- Esperamos que los ítems del área "Recursos personales, afrontamiento y resiliencia" se asocien positivamente con resiliencia y negativamente con ansiedad y depresión, salvo el ítem que evalúa esfuerzo percibido para hacer frente a la diálisis, con el que esperamos que las correlaciones sean en la dirección opuesta.

- Por otro lado, con respecto a los acontecimientos vitales difíciles (área "Acontecimientos vitales"), esperamos que las personas que los hayan experimentado muestren niveles mayores de depresión y ansiedad.

- Finalmente, esperamos que aquellos que indican que no se desaniman fácilmente, y que han sacado algo bueno de esta experiencia muestren mayor resiliencia y menor depresión. 


\section{Método}

Participantes

Los participantes de este estudio fueron 113 pacientes que estaban recibiendo tratamiento de HD en un hospital universitario y dos centros de diálisis de la Comunidad Valenciana. Los criterios de inclusión en el estudio fueron: a) ser mayor de 18 años; b) estar diagnosticado de ERCA; c) estar recibiendo tratamiento de diálisis; d) tener una adecuada comprensión del idioma y e) dar su consentimiento por escrito para ser evaluado. Los criterios de exclusión fueron: a) llevar menos de tres meses en el programa actual de diálisis; b) presentar deterioro cognitivo y c) padecer alguna enfermedad psiquiátrica activa que impida la correcta comprensión y respuesta de la entrevista o altere el estado de conciencia. La media de edad de los participantes fue de 63,35 años (DT=15,81), y el 66,4\% fueron varones. El $93,8 \%$ son de nacionalidad española. Con respecto a su estado civil, el $69 \%$ de los pacientes estaban casados o viviendo en pareja, el 13,3\% estaban solteros, el $13,3 \%$ estaban viudos, y el $4.4 \%$ estaban separados o divorciados. El $91,2 \%$ no se encontraba laboralmente activo en el momento de la evaluación El 56,6\% tenían estudios primarios, el $31 \%$ secundarios y el $12,4 \%$ universitarios.

\section{Procedimiento}

Los pacientes de este estudio transversal fueron evaluados a lo largo del año 2014 por una psicóloga entrenada en la sala de HD de los tres centros donde se realizó la recogida de datos. Tras informarles sobre el estudio y solicitar su consentimiento informado, todos los participantes respondieron a la herramienta CMAE-RD, y posteriormente completaron dos cuestionarios que evaluaban ansiedad, depresión y resiliencia, con propósitos de validación. La psicóloga evaluó a los pacientes durante las sesiones de HD y cada paciente tardo aproximadamente treinta minutos en completar la evaluación. La participación en el estudio fue voluntaria y se mantuvo la confidencialidad en todo el proceso de investigación.

\section{Materiales}

- Cuestionario Multidimensional de Adaptación a la Enfermedad para Pacientes Renales en Diálisis (CMAE-RD; Rodríguez-Rey ${ }^{(19)}$. Herramienta diseñada con el objetivo de facilitar y guiar una entrevista semiestructurada de evaluación a pacientes en diálisis, que incluyese los múltiples aspectos (necesidades y recursos) implicados en la adaptación a la ERCA. Consta de dos fases: Fase A y Fase B. La Fase A, que incluye 12 ítems, recoge toda la información de la historia médica y clínica y puede ser cumplimentada por el profesional o consultando al médico de referencia o la historia clínica del paciente. Esta fase incluye dos áreas "Datos Médicos" e "Historia Psicopatológica Previa". La Fase B, que incluye 51 ítems, está compuesta por nueve áreas que conforman la evaluación al paciente. Esta fase incluye las siguientes áreas: "Datos sociodemográficos" (6 ítems), "Información", (9 ítems), "Medicación y dieta" (3 ítems), "Apoyo social" (4 ítems), "Estado de ánimo" (2 ítems), "Recursos personales, afrontamiento y resiliencia" (8 ítems), "Aprendizaje y cambios percibidos" (5 ítems), "Espiritualidad" (2 ítems) y "Planificación de cuidados" (2 ítems). Las preguntas tienen cuatro formatos de 
respuesta; tres de ellos de carácter cerrado (respuestas dicotómicas, nominales categóricas, y escalas tipo Likert de 10 puntos), y uno de carácter abierto y cualitativo. La herramienta completa se encuentra recogida en el Anexo 1.

- Hospital Anxiety and Depression Scale (HADS) ${ }^{(24)}$. Consta de 14 ítems con formato de respuesta tipo Likert de 4 puntos ( 0 a 3 ) siete corresponden a la subescala de ansiedad (HADS-A) y los otros siete a la de depresión (HADS-D). La puntuación para cada subescala oscila entre 0 y 21 puntos. Ha mostrado tener adecuadas propiedades psicométricas en distintos grupos de pacientes, incluyendo enfermos renales en hemodiálisis ${ }^{(25)}$. Una revisión mostró que la media de alphas de Cronbach fue de 0,83 para la subescala HADS-A y.82 para la HADS-D ${ }^{(26)}$. Para ambas subescalas una puntuación inferior a 8 implica ausencia de depresión/ansiedad, una puntuación igual o superior a 11 indica un caso de ansiedad o depresión moderado, y una puntuación igual o superior a 15 indica un caso grave. En este estudio se ha empleado la versión Española ${ }^{(27)}$ que ha mostrado tener adecuada consistencia interna $(\alpha \text { entre } 0,80 \text { y } 0,87)^{(28)}$. En el presente estudio el índice de consistencia interna fue $\alpha=0,83$, para la escala global, $\mathrm{y}=0,76$ y 0,78 respectivamente para las subescalas HADS-A y HADS-D.

- Connor Davidson Resilience Scale-version de 2 items (CDRISC 2) ${ }^{(29)}$. Escala de evaluación de resiliencia, entendida como las cualidades personales que permiten enfrentarse exitosamente a situaciones amenazantes o potencialmente estresantes ${ }^{(30)}$. Esta versión de la escala está compuesta por dos ítems con cinco opciones de respuesta $(0=$ nunca; $5=$ caso siempre $)$. Puntuaciones más elevadas en esta escala indican niveles mayores de resiliencia. En la muestra de este estudio, la consistencia interna fue $\alpha=0,57$.

- Datos sociodemográficos: edad, género y nivel de estudios.

- Datos de perfil clínico: tiempo desde que recibió el diagnóstico, tiempo que lleva en diálisis en meses, forma de entrada en diálisis en el centro actual (urgente, programada, trasladado desde otro centro), presencia de algún trasplante renal previo, presencia de historia psicopatológica previa y consumo actual de psicofármacos. Además, se utilizaron la surprise question ("¿Le sorprendería que este paciente falleciera en el próximo año?”), que se considera un índice pronóstico fiable capaz de identificar a pacientes candidatos a cuidados paliativos ${ }^{(31)}$, y el índice de comorbilidad de Charlson ${ }^{32,33}$.

\section{Análisis de datos}

En primer lugar, se obtuvieron los estadísticos descriptivos de los datos clínicos de los pacientes evaluados. A continuación, con el objetivo de conocer en qué grado los pacientes puntuaban en las distintas áreas de la herramienta CMAE-RD (información, medicación y dieta, apoyo social, estado de ánimo, etc.) se obtuvieron los estadísticos descriptivos y frecuencias de dichas variables. Además, para analizar si había alguna diferencia en su estado de ánimo en función del centro en que se dializaba el paciente se realizó un análisis de varianza (ANOVA). También se calcularon las puntuaciones medias de los pacientes en ansiedad, depresión y resiliencia y el porcentaje de pacientes con depresión/ ansiedad ligera moderada y severa. Posteriormente, se realizó un análisis de fiabilidad de las diferentes áreas del CMAE-RD mediante alfa de Cronbach $(\alpha)$. 
Las evidencias de validez se han obtenido a través de distintos tipos de análisis. Para estudiar la validez relacionada con un criterio externo incluimos las correlaciones de Spearman entre algunos de los datos clínicos recogidos y los ítems del cuestionario para los datos cuantitativos, y diferencias de medias para los dicotómicos. A continuación, para estudiar la validez concurrente incluimos las correlaciones de Spearman entre las diferentes puntuaciones de los ítems del CMAE-RD y las de los cuestionarios CDRISC-2 y HADS. Además, utilizamos el estadístico t de Student para comparar las medias en ansiedad, depresión y resiliencia, en algunos ítems dicotómicos del CMAE-RD.

\section{Resultados}

\section{Datos clínicos}

Todos los pacientes evaluados estaban en tratamiento de HD. El tiempo medio desde el diagnóstico de ERCA en el momento de la evaluación fue de 75,08 meses (DT= $73,58)$. La media de tiempo que llevaban en $\mathrm{HD}$ fue de 50,10 meses $(\mathrm{DT}=54,02)$. Con respecto a cómo entraron en $\mathrm{HD}$ en el centro actual, el 64,6\% lo hicieron de forma programada desde consulta de ERCA, el 24,1\% inició diálisis de forma urgente, y el $10,7 \%$ fue trasladado desde otro centro. El 12,5\% había tenido un trasplante renal previo a la técnica actual de HD. La puntuación media en el índice de Charlson fue de 6,66 (DT=2,54). Para el 73,5\% de los pacientes, su nefrólogo ha respondido "sí" a la surprise question incluida en la Fase A del CMAE-RD, lo que implica que este porcentaje de la muestra no sería candidato a cuidados paliativos ${ }^{(31)}$. El $15,9 \%$ de los evaluados tiene historia de psicopatología previa, y el 47,7\% consume psicofármacos.

\section{Descriptivos puntuaciones herramienta CMAE-RD}

La Tabla 1 recoge los estadísticos descriptivos de las respuestas de los participantes a los ítems con formatos de respuesta dicotómica, nominal categórica, y escalas tipo Likert de 10 puntos del CMAE-RD. De entre los descriptivos recogidos en la Tabla 1, destaca el hecho de que menos del $50 \%$ de los participantes conociesen bastante o totalmente el diagnóstico. Sin embargo, más del 70\% indicaron que no necesitaban más información sobre ningún aspecto. Refirieron niveles elevados de adhesión y autonomía en la toma de medicación. Un 37,2\% refirió problemas con la dieta. En general, los pacientes indicaron un elevado nivel de satisfacción con su apoyo social (especialmente con sus familias y el personal sanitario), niveles bajos de ansiedad y tristeza, y elevada capacidad percibida para hacer frente a la diálisis. Las dimensiones en las que refirieron más cambios a consecuencia de la entrada en diálisis fueron sus emociones y su forma de ser. Más de la mitad de los pacientes indicaron que han sacado algo bueno de la enfermedad. Menos de un tercio refirieron tener información sobre las instrucciones previas. No se encontraron diferencias significativas en los niveles de tristeza y ansiedad en función del centro en que estaban recibiendo diálisis $(p>0,05)$. 
Tabla 1. Descriptivos de las respuestas de los participantes a los ítems cuantitativos y categóricos del CMAE-RD $(\mathrm{N}=113)$.

\begin{tabular}{|c|c|c|c|c|}
\hline \multicolumn{5}{|l|}{ Dimensión "Información" } \\
\hline & No & Ligeramente & Bastante & Totalmente \\
\hline Conoce diagnóstico (\%) & 17,7 & 35,4 & 21,2 & 25,7 \\
\hline Conoce tratamiento $(\%)$ & 6,2 & 24,8 & 29,2 & 39,8 \\
\hline \multirow[t]{2}{*}{ Conoce cuidados $(\%)$} & 0 & 23 & 32,7 & 44,2 \\
\hline & Sí & & No & \\
\hline Necesita más información (\%) & 22,1 & & 77,9 & \\
\hline \multirow[t]{2}{*}{ Autónomo toma medicación (\%) } & 82,3 & & 17,7 & \\
\hline & & $\mathrm{Si}$ & A medias & No \\
\hline \multicolumn{2}{|c|}{ Conoce medicación que toma su función (\%) } & 72,6 & 24,8 & 2,7 \\
\hline Nivel de comprensión (0-10) & \multicolumn{2}{|c|}{$7,90(D T=1,89)$} & & \\
\hline Percepción participación $(0-10)$ & \multicolumn{2}{|c|}{$6,56(D T=3,5)$} & & \\
\hline Deseo de participación (0-10) & \multicolumn{2}{|c|}{$5,18(D T=3,51)$} & & \\
\hline
\end{tabular}

\section{Dimensión "Medicación y dieta"}

Toma medicación $(0-10) \quad 9,68(, 85)$

\begin{tabular}{lcc}
\hline & $\mathrm{Si}$ & No \\
\hline Dificultad medicación (\%) & 17,7 & 82,3 \\
Dificultad dieta (\%) & 37,2 & 62,8 \\
\hline
\end{tabular}

\section{Dimensión "Apoyo social"}

Satisfacción familiar (0-10)

9,34 $(D T=1,84)$

Satisfacción amigos/vecinos (0-10)

7,85 $(D T=3,24)$

Satisfacción profesionales (0-10)

$9,39(D T=1,07)$

\begin{tabular}{lcc}
\hline & $\mathrm{Si}$ & No \\
\hline $\begin{array}{l}\text { Alguien puede hacer algo para que } \\
\text { se sienta mejor (\%) }\end{array}$ & 12,4 & 87,6 \\
\hline
\end{tabular}

\section{Dimensión "Estado de ánimo"}

\begin{tabular}{lcc}
\hline Tristeza (0-10) & $2,69(D T=2,80)$ & \\
Ansiedad (0-10) & $2,34(D T=2,71)$ & \\
\hline Dimensión "Eventos vitales" & & No \\
\hline & $\mathrm{Si}$ & 68,1 \\
\hline $\begin{array}{l}\text { Acontecimiento difícil en los últimos } \\
\text { seis meses (\%) }\end{array}$ & 31,9 & \\
\hline
\end{tabular}




\section{Dimensión "Recursos personales, afrontamiento y resiliencia"}

\begin{tabular}{|c|c|c|c|c|c|}
\hline Esfuerzo diálisis (0-10) & \multicolumn{5}{|c|}{$2,85(D T=2,96)$} \\
\hline Capaz de afrontar diálisis $(0-10)$ & \multicolumn{5}{|c|}{$8,84(D T=1,62)$} \\
\hline \multirow[t]{2}{*}{ Control percibido $(0-10)$} & \multicolumn{5}{|c|}{$5,21(D T=3,91)$} \\
\hline & & $\mathrm{Si}$ & & & \\
\hline \multirow[t]{2}{*}{ Desanima fácilmente (\%) } & \multicolumn{3}{|c|}{35,4} & \multicolumn{2}{|c|}{64,6} \\
\hline & M, bien & Bien & Regular & Mal & Muy mal \\
\hline $\begin{array}{l}\text { Percepción entorno } \\
\text { afrontamiento }(\%)\end{array}$ & 52,7 & 33,9 & 11,6 & 1,8 & 0 \\
\hline $\begin{array}{l}\text { Percepción personal } \\
\text { afrontamiento }(\%)\end{array}$ & 32,1 & 52,7 & 9,8 & 4,5 & 0,9 \\
\hline
\end{tabular}

\section{Dimensión "Espiritualidad"}

\begin{tabular}{lcc}
\hline & Si & No \\
\hline Creencia espiritual/religiosa (\%) & 63,7 & 36,3 \\
\hline Grado en que ayuda (0-10) & \multicolumn{3}{c}{$6,77(D T=3,48)$} \\
\hline
\end{tabular}

\section{Dimensión "Aprendizaje y cambio"}

\begin{tabular}{lcc}
\hline & $\mathrm{Si}$ & No \\
\hline Cambio emociones (\%) & 46 & 54 \\
Cambio forma de ser (\%) & 49,6 & 50,4 \\
Cambio relaciones con otros (\%) & 20,4 & 79,6 \\
Cambio creencias (\%) & 8 & 92 \\
Sacado algo bueno (\%) & 56,6 & 43,4 \\
\hline
\end{tabular}

Dimensión "Planificación de cuidados"

\begin{tabular}{lll}
\hline Conocimiento IP (\%) & 26,5 & 73,5 \\
Desea información IP (\%) & 30,1 & 69,9
\end{tabular}

Nota: $\mathrm{DT}=$ desviación típica; $\mathrm{IP}=$ instrucciones previas

Niveles de ansiedad, depresión y resiliencia

La media en la subescala HADS-A fue de 4,04 (DT=3,40), y en HADS-D de 4,09 $(D T=3,76)$, siendo para ambas el posible rango de puntuaciones de entre 0 y 21 . Los niveles de ansiedad y depresión calculados a partir de los puntos de corte de dicha escala se encuentran en la Tabla 2 [Insertar]. El 13,3\% mostró niveles al menos leves de ansiedad, y el 18,6\% de depresión. La puntuación media en resiliencia cuyo rango posible se encontraba entre 1 y 8 puntos fue de $7(D T=1,63)$. 
Tabla 2. Niveles de ansiedad y depresión según los puntos de corte del cuestionario HADS $(\mathrm{N}=113)$.

\begin{tabular}{llcc}
\hline & & $n$ & Porcentaje (\%) \\
\hline Ansiedad (HADS-A) & No ansiedad $(<8)$ & 98 & 86,7 \\
& Caso leve $(8-10)$ & 10 & 8,8 \\
& Caso moderado $(11-14)$ & 3 & 2,7 \\
& Caso grave $(15-21)$ & 2 & 1,8 \\
\hline Depresión (HADS-D) & No depresión $(<8)$ & 92 & 81,4 \\
& Caso leve $(8-10)$ & 15 & 13,3 \\
& Caso moderado $(11-14)$ & 4 & 3,5 \\
& Caso grave $(15-21)$ & 2 & 1,8 \\
\hline
\end{tabular}

Índices de fiabilidad

Dado que varias de las áreas del cuestionario evaluaban cuestiones heterogéneas, aunque relativas a un mismo ámbito (por ejemplo, en el área "medicación y dieta" se evalúa tanto percepción de adhesión, como dificultades con la misma), consideramos que no es pertinente calcular la consistencia interna de todas las áreas y nos limitamos a calcular la de las siguientes: información (9 ítems), estado de ánimo (2 ítems) y recursos personales, afrontamiento y resiliencia (6 ítems). Obtuvimos unos Alphas de Cronbach de 0,70, 0,62 y 0,53 respectivamente.

\section{Validez relacionada con un criterio externo}

Para comprobar las evidencias de validez de los ítems de la Fase B del CMAE-RD, calculamos las correlaciones de las puntuaciones en algunos de sus ítems con los siguientes indicadores clínicos y demográficos incluidos en la Fase A del CMAE-RD: el índice de comorbilidad de Charlson ${ }^{(32,33)}$, el índice pronóstico surprise question ${ }^{(31)}$, presencia de historia psicopatológica previa, edad y nivel de estudios.

Índice de comorbilidad de Charlson: Encontramos que el índice de Charlson correlaciona de forma significativa y negativa con nivel de conocimiento sobre el diagnóstico $\left(r_{s}=-0,26, p=0,05\right)$, sobre el tratamiento $\left(r_{s}=-0,36, p<0,001\right)$, y sobre los cuidados (pastillas y dieta) $\left(r_{s}=-0,33, p<0,001\right)$. No se ha encontrado relación significativa con autonomía en la toma de la medicación.

Surprise question. Encontramos niveles mayores de tristeza en los casos en que la respuesta a la surprise question ha sido afirmativa $(p=0,045)$ y de control percibido $(p=0,03)$ pero no diferencias en ansiedad.

Historia psicopatológica previa: Encontramos que las personas con historia de psicopatología tienen niveles mayores de tristeza $(p=0,05)$ pero, en contra de lo esperado, refieren niveles mayores de adherencia $(p=0,05)$.

Edad: Encontramos que edad correlaciona negativamente con conocimiento sobre el diagnóstico $\left(r_{s}=-0,52, p<0,001\right)$, conocimiento sobre el tratamiento $\left(r_{s}=\right.$ 
-.60; $p<0,01)$, conocimiento sobre la medicación y la dieta $\left(r_{s}=-0,425 ; p<0,001\right)$, deseo de participar en las tomas de decisiones $\left(r_{s}=-0,38 ; p<0,001\right)$, participación percibida $\left(r_{s=} 0240 ; p=0,01\right)$, y autonomía en la toma de la medicación $\left(r_{s}=\right.$ $-0,314 ; p=0,001)$.

Nivel de estudios: Encontramos que nivel de estudios correlaciona de forma significativa con nivel de conocimiento sobre el diagnóstico $\left(r_{s}=0,27, p=0,004\right)$, sobre la diálisis $\left(r_{s}=0,40, p<0,001\right)$ y sobre la medicación y la dieta $\left(r_{s}=0,31\right.$, $p=0,001)$.

En conjunto, estos análisis arrojan evidencias a favor de la validez relacionada con criterio externo de las áreas "Información" y "Estado de ánimo".

\section{Validez concurrente}

Para estudiar la validez concurrente de los ítems del CMAE-RD, calculamos correlaciones con las puntuaciones de las escalas HADS y CDRISC-2. Las correlaciones se muestran en la Tabla 3. Tal y como muestra la Tabla 3, la mayor parte de las relaciones se produjeron en la dirección esperada, lo que aporta evidencias a favor de la validez convergente de la escala.

Tabla 3. Correlaciones de los ítems de apoyo social, estado de ánimo y recursos personales, afrontamiento y resiliencia con las puntuaciones del CDRISC-2 (resiliencia) y e HADS (depresión y ansiedad).

\begin{tabular}{lccc}
\hline & Resiliencia & Ansiedad & Depresión \\
\hline Satisfacción con el apoyo familiar &, 085 &,- 138 &,- 182 \\
Satisfacción con apoyo de amigos/ vecinos &, $217^{*}$ &,$- 246^{* *}$ &,- 323 \\
Satisfacción con el equipo asistencial &, 114 &,- 058 &,- 109 \\
Tristeza &,$- 334^{* * *}$ &, $409^{* * *}$ &, $574^{* * *}$ \\
Ansiedad &,$- 297^{* * *}$ &, $580^{* * *}$ &, $438^{* * *}$ \\
Esfuerzo percibido &,$- 253^{* * *}$ &, $244^{* * *}$ &, $383^{* * *}$ \\
Capacidad percibida &, $353^{* * *}$ &,$- 260^{* *}$ &,$- 427^{* * *}$ \\
Control percibido &, $275^{* *}$ &, 068 &,$- 186^{*}$ \\
Percepción de afrontamiento del entorno &, $288^{* *}$ &,- 087 &,$- 379^{* * *}$ \\
Auto- percepción de afrontamiento &, $393^{* * *}$ &,- 194 &,$- 417^{* * *}$ \\
\hline
\end{tabular}

${ }^{*} \mathrm{p} \leq 0,05 ;{ }^{* *} \mathrm{p} \leq 0,01 ;{ }^{* * *} \mathrm{p} \leq 0,001$

Los resultados, mostrados en la Tabla 4, muestran que las diferencias se producen en la dirección esperada. 
Tabla 4. T de Student para comparar niveles de ansiedad, depresión y resiliencia en los ítems "acontecimiento difícil", "se desanima fácilmente" y "considera que ha sacado algo bueno de esta experiencia".

\begin{tabular}{lcccccc}
\hline & & N & $M$ & $D T$ & $t$ & $p$ \\
\hline Ansiedad (HADS-A) & & & & & & \\
\hline Acontecimiento difícil & si & 36 & 5,19 & 4,01 & 2,54 &, 013 \\
& no & 77 & 3,49 & 2,95 & & \\
\hline Depresión (HADS-D) & & & & & & \\
\hline Acontecimiento difícil & si & 36 & 5,44 & 4,44 & 2,69 &, 008 \\
& no & 77 & 3,45 & 3,23 & &, 001 \\
\hline Desanima fácilmente & si & 40 & 5,75 & 4,05 & 3,45 &, 01 \\
& no & 73 & 6,38 & 3,27 & & \\
\hline Sacado algo bueno & si & 64 & 3,30 & 3,39 & $-2,63$ & \\
& no & 49 & 5,12 & 3,99 & &, 011 \\
\hline Resiliencia (CDRISC-2) & & & & & & \\
\hline Desanima fácilmente & si & 40 & 5,57 & 1,69 & $-2,58$ & \\
& no & 73 & 6,38 & 1,53 & & \\
\hline Sacado algo bueno & si & 64 & 6,38 & 1,44 & 2,10 & \\
& no & 49 & 5,73 & 1,80 & &
\end{tabular}

\section{Discusión}

Este trabajo tenía como principal objetivo el estudio de las propiedades psicométricas de un cuestionario multidimensional de adaptación a la enfermedad para pacientes ERCA en diálisis. Dicha herramienta se concibió como un instrumento que facilitara y guiara una entrevista semiestructurada de evaluación de recursos y necesidades relacionados con su adaptación a la ERCA, de la misma manera que el cuestionario del que se deriva (el CMAE-OH) lo hacía con pacientes oncohematológicos.

Empezando por la fiabilidad, se han obtenido índices de entre 0,53 y 0,70 que, si bien no son muy elevados, pueden considerarse suficientes teniendo en cuenta las características de este instrumento (múltiples formatos de respuesta, escaso número de ítems en algunas dimensiones, etc.). Estos niveles son similares a los obtenidos en el estudio de Rodríguez-Rey et al. ${ }^{(19)}$ que describe el desarrollo de esta escala y sus propiedades psicométricas preliminares. Con respecto a la validez, encontramos diversos indicadores de validez externa y concurrente, existiendo evidencia especialmente fuerte para las áreas "Información" y "Estado de ánimo". Podemos concluir, por tanto, que en términos generales los ítems del CMAE-RD presentan niveles adecuados de fiabilidad y validez.

El CMAE-RD es la primera herramienta específicamente diseñada en castellano para evaluar distintas dimensiones psicológicas implicadas en la adaptación 
psicológica a la ERCA. Esta herramienta concuerda con la idea de que, por su complejidad, la evaluación psicológica al paciente crónico requiere de una perspectiva multidimensional ${ }^{(20,34)}$. El CMAE-RD tiene múltiples ventajas desde el punto de vista clínico, pues permite la evaluación de diferentes áreas de forma simultánea, de modo que puede servir como herramienta de cribado y como guía para la intervención. Además, cumple todos los requisitos establecidos por Arranz y Ulla ${ }^{(20)}$ para una herramienta multidimensional de evaluación de necesidades y recursos en pacientes con enfermedad: 1) brevedad y sencillez, 2) posibilidad de aplicarse mediante entrevista semiestructurada, 3) que proporcione datos útiles para docencia e investigación y 4) que este avalado por análisis sistemáticos que refrenden su calidad. A pesar de las ventajas de esta herramienta, somos conscientes de sus limitaciones en términos psicométricos, pues las mismas características que lo hacen adecuado para la práctica clínica, conducen a que su validez y fiabilidad sea limitada.

Queda para futuros estudios la tarea de someter a contrastación su sensibilidad al cambio y su nivel de fiabilidad test-retest, así como la obtención de puntos de corte para los dos ítems que evalúan estado de ánimo, tal y como se ha realizado en el instrumento EE-D, que fue diseñado para ser empleado por profesionales de enfermería ${ }^{(15,16)}$. Por otro lado, sería interesante adaptarlo a pacientes renales estudiar $\mathrm{su}$ funcionamiento en pacientes sometidos a otro tipo de tratamiento (diálisis peritoneal hemodiálisis domiciliaria, trasplante de donante vivo o cadáver), así como adaptarlo a estadíos más precoces de la enfermedad (previos a la necesidad de diálisis) para explorar su funcionamiento como detector de conductas de no adhesión.

Pese a las limitaciones de este trabajo, consideramos que supone un paso importante en la atención integral de los pacientes renales en diálisis, ya que proporciona a los psicólogos una herramienta útil desde el punto de vista clínico para evaluar necesidades y recursos en pacientes en diálisis.

\section{Conclusiones}

La herramienta CMAE-RD puede facilitar el punto de partida que permita guiar una intervención individualizada, llevada a cabo por un/a psicólogo/a y destinada a facilitar la adaptación del paciente a la enfermedad renal y al tratamiento de diálisis.

Los avances en la detección precoz y los tratamientos del cáncer han reducido significativamente la mortalidad asociada a este diagnóstico, haciendo más importante tanto el seguimiento a medio-largo plazo de los pacientes, como la promoción de su adaptación a unas condiciones de salud que, en ocasiones, son crónicas ${ }^{(35)}$. Este hecho, junto al reconocimiento de la importancia de individualizar el tratamiento psicológico para cada paciente, promovió el desarrollo de instrumentos de evaluación como el CMAE-OH de Arranz y Ulla ${ }^{(20)}$. El hecho de que el CMA-RD de Rodríguez-Rey et al. ${ }^{(19)}$ se haya adaptado del contexto de la onco-hematología muestra que los desarrollos y avances producidos en el ámbito de la psicooncología son extrapolables a otras áreas de la Psicología de la Salud.

A pesar de que la psicooncología como disciplina específica tuvo un nacimiento relativamente tardío, pues se empezó a desarrollar en el último cuarto del pasado siglo, su avance desde entonces ha sido muy notable tanto en el ámbito clínico como de investigación ${ }^{(36)}$. Esperamos que este trabajo contribuya a que la psiconefrología, 
área actualmente en desarrollo ${ }^{(37)}$, continúe constituyéndose como un ámbito de conocimiento específico, con sus propias aportaciones teórico-prácticas, áreas de acción, retos y desafíos.

\section{Agradecimientos}

A Eva María Jareño y a Pablo Molina por su apoyo durante el proceso de recogida de datos.

\section{Referencias bibliográficas}

1. Levey AS, Coresh J. Chronic kidney disease. Lancet 2012;14,379:165-80. doi: 10.1016/ S0140-6736(11)60178-5.

2. Gorostidi M, Sánchez-Martinez M, Ruilope LM, Graciani A, de la Cruz JJ, Santamaría R, del Pino MD, Guallar-Castillón P, de Álvaro F, Rodriguez-Artalejo F, Banegas JR. Prevalencia de la enfermedad renal crónica en España: impacto de la acumulación de factores de riesgo cardiovascular. Nefrología 2018;38,6:573-680. doi: 10.1016/j. nefro.2018.04.004

3. Weisbord SD, Fried LF, Arnold RM, Fine MJ, Levenson, DJ, Peterson RA, Switzer GE. Prevalence, severity, and importance of physical and emotional symptoms in chronic hemodialysis patients. J Am Soc Nephro 2005;16:2487-94. doi: 10.1681/ ASN.2005020157

4. Finkelstein FO, Finkelstein SH. Health related quality of life and the CKD patient: Challenges for the nephrology community. Kidney Int 2009;76: 946-52. doi:10.1038/ ki.2009.307

5. Rodríguez-Fructuoso M, Castro R, Oliveira I, Prata C, Morgado T. Quality of life in chronic kidney disease. Nefrología 2011; 31: 91-6. doi: 10.3265/Nefrologia.pre2010. Jul.10483

6. Feroze U, Martin D, Reina-Patton A, Kalantar-Zadeh K, Kopple JD. Mental health, depression, and anxiety in patients on maintenance dialysis. Iran J Kidney Dis 2010;4:173-80.

7. Vasilopoulou C, Bourtsi E, Giaple S, Koutelekos I, Theofilou P, Polikandrioti M. The Impact of Anxiety and Depression on the Quality of Life of Hemodialysis Patients. Glob. J. Health Sci 2016;8:45-55. doi: 10.5539/gjhs.v8n1p45

8. Watnick S, Kirwin P, Mahnensmith R, Concato J. The prevalence and treatment of depression among patients starting dialysis. Am J Kidney Dis 2003;41:105-10. doi: doi: 10.1053/ajkd.2003.50029

9. Chen CK, Tsai YC, Hsu HJ, Wu IW, Sun CY, Chou CC, Lee CC, Tsai CR, Wu MS, Wang LJ. Depression and suicide risk in hemodialysis patients with chronic renal failure. Psychosomatics 2010;51:528-528.e6. doi: 10.1176/appi.psy.51.6.528.

10. Cukor D, Coplan J, Brown C, Friedman S, Newville H, Safier M, Spielman LA, Peterson RA, Kimmel PL. Anxiety disorders in adults treated by hemodialysis: a single-center study. Am J Kidney Dis 2008;52:128-36.

11. Callahan D. Death and the research imperative. N Engl J Med 2000;342:654-6. doi: 10.1056/NEJM200003023420910 
12. Larsson EB, Yao X. Clinical empathy as emotional labor in the patient-physician relationship. JAMA 2005;293:1100-6. doi:10.1001/jama.293.9.1100

13. Santacruz PL, Rangel ME, Navas N, Bolívar Z. La visión integradora biopsicosocial como estrategia ante el paciente con enfermedad renal crónica. Requisito contemporáneo. Nefrología 2005;26:635-6.

14. Paparrigopoulos T, Theleritis C, Tzavara C, Papadaki A. Sleep disturbance in haemodialysis patients is closely related to depression. Gen Hosp Psychiatry 2009;31, 2:175-7. doi:10.1016/j.genhosppsych.2008.09.016

15. García-Llana H, Rodríguez-Rey R, Rollán, MJ, Dapena Vielba F, Ramos F, Trocoli, F, Selgas S. Desarrollo de un instrumento para la evaluación del malestar emocional para pacientes renales en diálisis. Enferm Nefrol 2016;19:349-57. doi: 10.4321/S225428842016000400006

16. Rodríguez-Rey R, García-Llana H, Ruiz-Álvarez MP, Gómez-Gómez A, del Peso G, Selgas R. Multicenter validation of the emotional state instrument for dialysis patient. Nur Res 2019;68:39-47. doi: 10.1097/NNR.0000000000000321

17. García-Llana H, Barbero J, Olea T, Jiménez C, del Peso G, Miguel JL, Sánchez R, Celadilla O, Trocoli F, Argüello MT, Selgas R. Incorporación de un psicólogo en un servicio de nefrología: criterios y proceso. Nefrología 2010;30:297-330.

18. Monica RM, Delli Zotti GB, Spotti D, Sarno L. L'inserimento della figura dello psicologo all'interno dell'Unità Operativa di Nefrologia-Dialisi-Ipertensione: dalla valutazione dei bisogni alla definizione di un modello d'intervento [Integration of a psychologist into Nephrology-Dialysis-Hypertension Operative Unit: from needs evaluation to the definition of an intervention model]. G Ital Nefrol 2014; 31; 1-17

19. Rodríguez-Rey R, García-Llana H, Barbero J, Bajo MA, Sánchez Villanueva R, del Peso $\mathrm{G}$, et al. propiedades psicométricas preliminares de un Cuestionario Multidimensional de Adaptación a la Enfermedad para Pacientes Onco-Hematológicos (CMAE-OH) adaptado a pacientes renales en diálisis (CMAE-RD). Psicooncología 2012;9:369-91. doi: 10.5209/ rev_PSIC.2013.v9.n2-3.40907

20. Arranz P, Ulla S. Diseño y validación de un cuestionario multidimensional de adaptación a la enfermedad para pacientes oncohematológicos (CMAE-OH). Psicooncología 2008;5:7-25.

21. Costa M, López E. Educación para la salud. Una estratega para cambiar los estilos de vida. Madrid: Pirámide; 1996.

22. Albee GW. A competency model to replace the defect model. En Gibbs MS, Lachenmeyer JP, Sigal J, editors. Community Psychology. Nueva York: Gardner Press; 1980. P.213-38.

23. Lazarus RS, Folkman S. Stress, Appraisal and Coping. Nueva York: Springer Publishing; 1984.

24. Zigmond AS, Snaith RP. The hospital anxiety and depression scale. Acta Psychiatr Scand 1983;67:361-370.

25. Untas A, Aguirrezabal M, Chauveau P, Leguen E, Combe C, Rascle N. Anxiety and depression in hemodialysis: Validation of the Hospital Anxiety and DepressionS (HADS). Nephrol Ther 2009;5:193-200. doi: 10.1016/j.nephro.2009.01.007

26. Bjelland I, Dahl AA, Haug TT, Neckelmann D. The validity of the hospital anxiety and depression scale: An updated literature review. J Psychosom Res 2002;52:69-77. doi: 10.1016/s0022-3999(01)00296-3

27. Quintana JM, Padierna A, Esteban C, Arostegui I, Bilbao A, Ruiz I. Evaluation of the Psychometric characteristics of the Spanish version of the Hospital Anxiety and Depression Scale. Acta Psychiatr Scand 2003;10:216-21. doi: 10.1034/j.1600-0447.2003.00062.x 
28. Terol-Cantero MC, Cabrera-Perona V. Hospital anxiety and depression scale (HADS) review in Spanish samples. An Psicol 2015;31: 494-503. doi:10.6018/ analesps.31.2.172701. doi: 10.6018/analesps.31.2.172701

29. Vaishnavi S, Connor K, Davidson JRT. An abbreviated version of the Connor-Davidson Resilience Scale (CD-RISC), the CD-RISC2: Psychometric properties and applications in psychopharmacological trials. Psychiatry Res 2007;152:293-7.

30. Connor KM, Davidson JRT. Development of a new resilience scale: the Connor-Davidson Resilience Scale (CD-RISC). Depress Anxiety 2003;18:71-82. doi:10.1002/da.10113

31. Moss AH, Ganjoo J, Sharma S, Gansor J, Senft S, Weaner B, et al. Utility of the "Surprise" Question to Identify Dialysis Patients with High Mortality. Clin J Am Soc Nephrol 2008;3:1379-84. doi: 10.2215/CJN.00940208.

32. Charlson ME, Pompei P, Ales KL, MacKenzie CR. A new method of classifying prognostic comorbidity in longitudinal studies: development and validation. J. Chronic Dis $1987 ; 40: 373-83$.

33. Charlson ME, Charlson RE, Paterson JC, Marinopoulos SS, Briggs WM, Hollenberg JP. The Charlson comorbidity index is adapted to predict costs of chronic disease in primare care patients. J. Clin. Epidemiol 2008;61: 1234-40. doi: 10.1016/j.jclinepi.2008.01.006.

34. Velikova G, Booth L, Smith AB, Brown PM, Lynch P, Brown JM, Selby PJ. Measuring quality of life in routine oncology practice improves communication and patient wellbeing: a randomized controlled trial. J Clin Oncol 2004;15, 22:714-24. doi: 10.1200/ JCO.2004.06.078

35. López-Fernández T, Martín García A, Santaballa Beltrán A, Montero Luis Á, García Sanz $\mathrm{R}$, Mazón Ramos P, et al. Cardio-Onco-Hematología en la práctica clínica. Documento de consenso y recomendaciones. Rev Esp Cardiol 2017;70(6):474- 86.

36. Holland JC, editor. Psycho-oncology. Oxford University Press, 2010.

37. Novak M. Psychonephrology: An Emerging Field. Prim. Psychiatry 2008;15:43-4 